\title{
Gamma-ray Imaging System with a Rotation Collimator
}

\author{
Nam Young KIM and Jang Ho HA \\ Korea Atomic Energy Research Institute, 1045 Daedeokdaero, Daejon, 305-353, Korea
}

\begin{abstract}
The performance of a gamma-ray imaging system consisting of a gridded collimator and coaxial NaI(Tl) detector was investigated. A gamma-ray imaging system with rotation collimators does not require a position-sensitive detector so as to find the location of gamma-ray sources and therefore this feature allows for significant amount of flexibility not only detector selection but also the geometry of the system. A reconstructed image from the ${ }^{137} \mathrm{Cs}$ gamma-ray source have been obtained by using the simple flat-fielded back-projection algorithm. The background events are jumbled together in the correct modulation-patterns. Therefore a possibility may be caused by those events that the image is reconstructed at random on the image plane. The theoretical image resolution for the current proof-of-principle gamma-ray imager is $\sim 1^{\circ}$ with a FOV $\sim 53.6^{\circ}$. The image resolution with FWHM shows about $11.2^{\circ}$. Therefore our gamma-ray imaging system is required the more collimators, because the reconstructed image was significantly affected by the modulation patterns. Future research will be to focus on the improvement of the image resolution with two or more collimators.
\end{abstract}

KEYWORDS: modulation pattern, image reconstruction, NaI(Tl) detector, gridded collimator

\section{Introduction}

Rotating modulation collimators (RMCs) are one of the techniques for gamma-ray imaging using a mechanical collimator. Unlike pin-hole collimator or coded aperture, RMCs make with a single-channel detector to record a timedependent modulation-pattern that involves a location information of interest gamma-ray sources. A basic RMC consists of a detector located beneath a gridded collimator. Currently, the RMC method is using to get a reconstructed image of the sun in the Reuven Ramaty High Energy Solar Spectroscopic Imager (RHESSI) and the RHESSI allowed for excellent performance of the infinite focus of solar imaging. The gammaray imaging system using RMCs has been studied for astronomical, medical application, and homeland security. ${ }^{1-4)}$

The greatest advantage of the RMC imager is that this system does not requires a position-sensitive detector so as to find the location of the gamma-ray source and their ability to achieve good image resolution over a relatively wide field-of-view (FOV). ${ }^{3)}$ Therefore this feature allows for significant amount of flexibility not only detector selection but also the geometry of the system. And this system is a nonspectroscopic system, the data acquisition systems can be made relatively simple depending on the objective.

Many assumptions that are valid for a focus of infinity such as RHESSI are no longer vaild in the near-field environment so that the incident gamma-ray as not parallel. ${ }^{4}$ To that reason, we have designed and tested the proof-of-principle system for the gamma-ray as well as neutron imaging in the future.

\section{Principle of Image Reconstruction}

The rotating collimator temporally modulates the intensity of the gamma-ray flux transmitted to a detector. Thus the rotation causes the transmission of the grid viewing a gamma-ray

\footnotetext{
Corresponding author, E-mail: jhha@kaeri.re.kr
}

c Atomic Energy Society of Japan source that was a modulated count-rates; the modulation pattern depends on the location of the source in the FOV. The image resolution $(\Delta \theta)$ and FOV for the gamma-ray imaging system are defined $\mathrm{as}^{3)}$ :

$$
\begin{aligned}
\Delta \theta & =\tan 1\left(\frac{0.5 p}{D_{d}}\right) \\
F O V & =\frac{R_{c}+R_{d}}{D_{d}}
\end{aligned}
$$

where $p$ is the width of grid-pitch, $D_{d}$ is the distance between collimator and detector, and, $R_{c}$ and $R_{d}$ is the radius of detector and collimator, respectively.

Because the measured data is only an indirect measurement of the image, a suitable treatment of the modulated countrates produces an image in gamma-rays. The ideal modulation function $I_{m}(\theta)$ means the probability of detecting counts from a point source located at position $m$, when the collimator is at angle $\theta^{5,6)}$ :

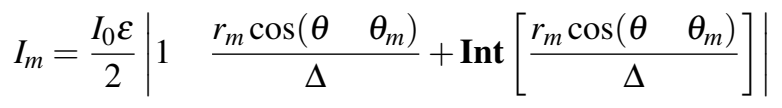

where $\varepsilon$ is the detection efficiency, $\operatorname{Int}[x]$ is the integer which satisfies $\operatorname{Int}[x] \leqslant x<\operatorname{Int}[x+1]$ and $0 \leqslant \theta<\pi, I_{0}$ is the incident flux of the gamma-ray source in count $/ \mathrm{cm}^{2} / \mathrm{sec}$ , and $\Delta$ is grid pitch of the collimator.

For image reconstruction, we are currently employing an intermediate image which is called the simple flat-fielded back-projection algorithm. The modulation patterns $I_{m}(\omega t)$ are calculated and then added on the image voxel $m$ every time $t$ with angular velocity $\omega$ of the collimator. The simple flatfielded back-projection algorithm has been of the form ${ }^{7)}$ :

$$
S_{m}=\frac{\sum_{t} O(t)\left(I_{m}(\omega t)<I_{m}>\right)}{A T\left(<I_{m}^{2}><I_{m}>^{2}\right)}
$$


where $S_{m}$ is the estimated probability that location of gamma-ray source is the $m$ th voxel, $O(t)$ represents the observed counts at time $t, A$ is the effective area of the detector, and, $T$ is the total livetime in seconds.

Therefore, reconstruction of images required a knowledge of $I_{m}(\omega t)$ for the gridded collimators as a function energy and direction of the incidence since the image sensitivity is proportional to the variance of the ideal modulation function for each image voxel.

\section{Gamma-ray Imaging System}

Figure 1 shows a photograph of the our gamma-ray imaging system. Gamma-ray imaging system consisting of a gridded collimator and coaxial $\mathrm{NaI}(\mathrm{Tl})$ detector manufactured by Bicron. The purpose of the data acquisition system is to record the time and number of each gamma-ray detected, allowing the modulated count-rates to be determined as a function of rotation angle. Since RMC imaging system does not requires a position-sensitive detector, the $\mathrm{NaI}(\mathrm{Tl})$ detector have a suitable capabilities for the proof-of-principle system.

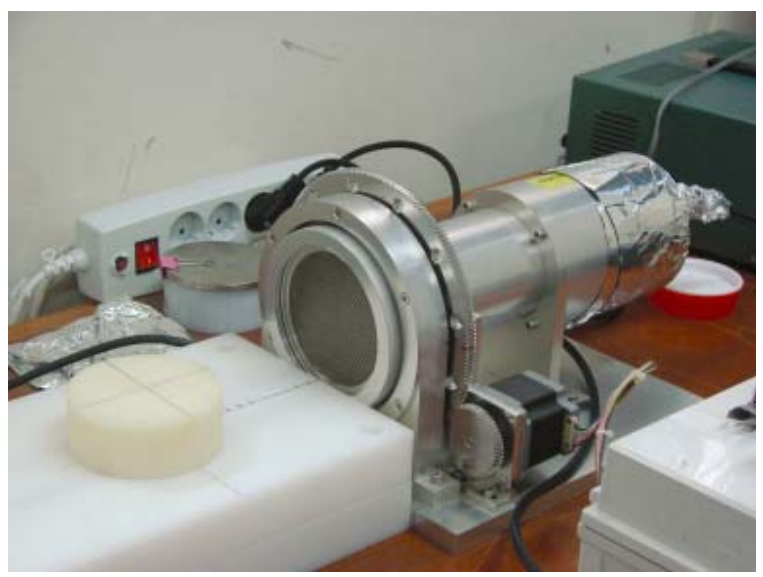

Fig. 1 Photograph of our Gamma-ray imaging system.

The $\mathrm{NaI}(\mathrm{Tl})$ crystal diameter and thickness are $76.2 \mathrm{~mm}$, equally. And the applied high-voltage was $+800 \mathrm{~V}$. The tungsten collimator has a diameter of $90.0 \mathrm{~mm}$ and 2.0 -mm-thick. The grid pitch and slit are $2 \mathrm{~mm}$ and $1 \mathrm{~mm}$ in width, respectively. The gridded collimator is mounted on the gear. The distance between collimator and detector is $55 \mathrm{~mm}$, and the angular velocity $\omega$ is setting up about 0.25 revolutions per minute by using stepping-motor control system. Hence, theoretical image resolution for the current proof-of-principle gamma-ray imager is $\sim 1^{\circ}$ with a FOV $\sim 53.6^{\circ}$.

\section{Experimental Results}

Figure 2 shows gamma-ray energy spectra of the $\mathrm{NaI}(\mathrm{Tl})$ detector for the standard gamma-ray source ${ }^{133} \mathrm{Ba}$ and ${ }^{137} \mathrm{Cs}$. A $\mathrm{NaI}(\mathrm{Tl})$ detector output was fed into a scintillation preamplifier (ORTEC-113) and then into a spectroscopy amplifier(ORTEC-672). The output of spectroscopy amplifier was read on a multichannel buffer (ORTEC-919) for the energy measurement. A background noise due to the steppingmoter control system of the collimator was picked up the pho- tomultiplier tube (PMT) base of the $\mathrm{NaI}(\mathrm{Tl})$ detector. Therefore, PMT base were enclosed in aluminum foil to protect against noise from the stepping-moter control system. The energy resolution was about $61.2 \mathrm{keV}$ in full width at half maximum (FWHM) at $661.6 \mathrm{keV}$ after channel calibration of multichannel buffer with gamma-ray peaks in a ${ }^{133} \mathrm{Ba}(81.0$ $\mathrm{keV}),{ }^{137} \mathrm{Cs}(661.6 \mathrm{keV})$, and, ${ }^{60} \mathrm{Co}(1173.2 \mathrm{keV}$ and 1332.5 $\mathrm{keV})$ source.
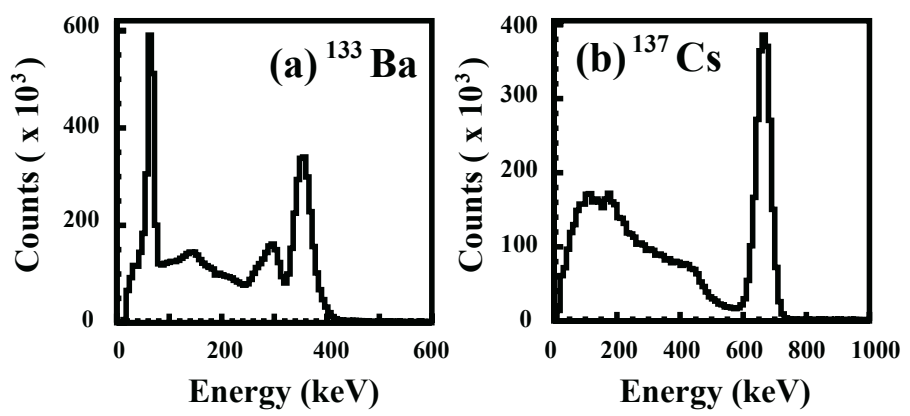

Fig. 2 Gamma-ray energy spectra with the present Gammaray imaging system for two gamma-ray sources, (a) ${ }^{133} \mathrm{Ba}$ and (b) ${ }^{137} \mathrm{Cs}$.

The experimental data for the image reconstruction recorded the counting numbers at one-second interval by using ORTEC-850 Quad Single-Channel Analyzer (SCA) and ULS-3000 4-Channel Counter/Timer/Rate Meter. The data acquisition software is a program offered by ULS Inc. and it is implemented and operated on a PC Window system.

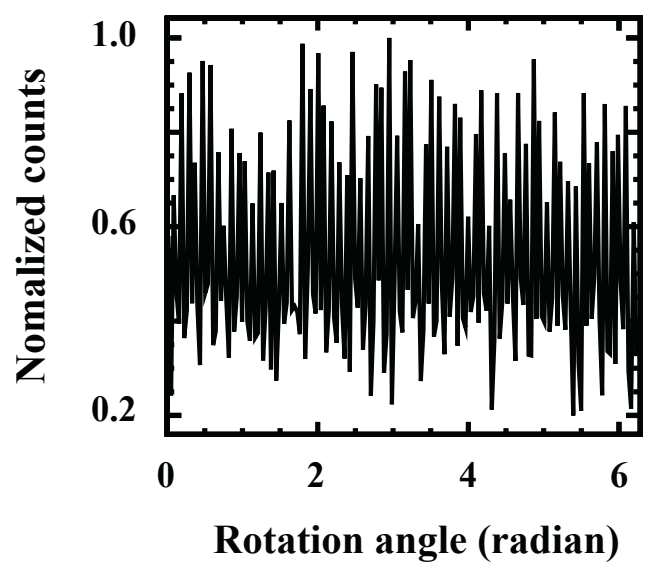

Fig. 3 Modulation patterns obtained by the our gamma-ray imaging system with the ${ }^{137}$ Cs gamma-ray source.

A $10 \mu \mathrm{Ci}{ }^{137} \mathrm{Cs}$ source were $100 \mathrm{~mm}$ from the gridded collimator and Figure 3 shows an experimental modulation pattern. However, because of the close proximity of the gammaray source or single collimator, there are such background events as the gamma-rays pass though without interaction with collimator or a grid space of collimator. Those events are jumbled together in the correct modulation-patterns. Therefore a possibility may be caused by those events that the image is reconstructed at random on the image plane. However, if the sum of the modulation patterns and background is sim- 
ilar to the $I_{m}(\omega t)$ at $r_{m} \sim 0$, such events are reconstructed on the central region of image plane.
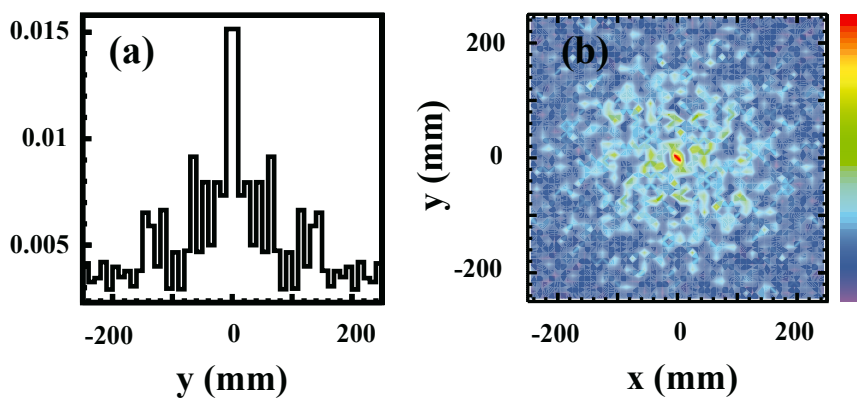

Fig. 4 The profile and two-dimensional image for a ${ }^{137} \mathrm{Cs}$ source obtained from the experimental data.

Figure 4 shows a profile and reconstructed image for gamma-ray source ${ }^{137} \mathrm{Cs}$. The image plane is perpendicular to the $z$-axis and $155.0 \mathrm{~mm}$ from the face of the detector and the size of image voxel is $10.0 \mathrm{~mm} \times 10.0 \mathrm{~mm}$. The $S_{m}$ in the Eq. (3) is calculated for each image voxel as the location of real gamma-ray source by using the ROOT software library. ${ }^{8)}$ Due to the geometry of the gridded collimator, the reconstructed image will not stand for a location of gamma-ray at the central extent around the image resolution in the Eq. (1) as well as the outside FOV of the gamma-ray imaging system. In order to evaluate the image resolution of the gamma-ray imaging system, the one-dimensional profile were selected in $\mathrm{a} \pm 10 \mathrm{~mm}$ on the $\mathrm{x}$-axis. The image resolution with FWHM shows about $11.2^{\circ}$. The reconstructed image is more blurred than the expected image resolution and the random images are reconstructed on the image plane because of the background events.

Consequently, the image reconstruction was significantly affected by the modulation patterns, this proof-of-principle system shows not enough to obtain an accurate location of the gamma-ray source with single collimator. Future research will be focus on the improvement of the image resolution with the two or more collimators.

\section{Summary}

The performance of a gamma-ray imaging system consisting of a gridded collimator and coaxial $\mathrm{NaI}(\mathrm{Tl})$ detector was investigated. A gamma-ray imaging system with rotation collimators does not require a position-sensitive detector so as to find the location of gamma-ray sources and therefore this feature allows for significant amount of flexibility not only detector selection but also the geometry of the system. A reconstructed image from the ${ }^{137} \mathrm{Cs}$ gamma-ray source have been obtained by using the simple flat-fielded back projection algorithm. The background events are jumbled together in the correct modulation-patterns. Therefore a possibility may be caused by those events that the image is reconstructed at random on the image plane. The theoretical image resolution for the current proof-of-principle gamma-ray imager is $\sim 1^{\circ}$ with a FOV $\sim 53.6^{\circ}$. The image resolution with FWHM shows about $11.2^{\circ}$. Therefore our gamma-ray imaging system is required the more collimators, because the reconstructed image was significantly affected by the modulation patterns. Future research will be to focus on the improvement of the image resolution with two or more collimators.

\section{Acknowledgment}

This work has been carried out under the nuclear R\&D program of the Ministry of Education, Science and Technology (MEST).

\section{References}

1) G. J. Hurford, et al., "The RHESSI imaging concept", Solar Physics, 210, 61 (2002).

2) D. M. Smith, et al., "Rotating modulation collimator imagers", New Astronomy Reviews, 48, 209 (2004).

3) B. R. Kowash, et al., "A rotating modulation imager for locating mid-range point source", Nuclear Instruments and Methods in Physics Reserch A 602, 477 (2009).

4) A. C. Sharma, et al., "Design and Construction of a prototype rotation modulation Collimator for near-field high-energy spectroscopic gamma Imaging", Nuclear Science Symposium Conference Record, 2006. IEEE M06-223, 2021 (2006).

5) V. Dadurkevičius and D. A. Ralys, "Modulation patterns of astronomical imaging systems based on rotating grids", Astrophysics and Space Science, 113, 233 (1985).

6) Y. Chen, et al., "Direct demodulation technique for rotating modulation collimator imaging", Astronomy \& Astrophysics Supplement Series, 128, 363 (1998).

7) P. Durouchoux, et al., "Gamma-ray imaging with a rotating modulator", Astronomy and astrophysics, 120, 150 (1983).

8) R. Brun and F. Rademakers, "ROOT-An object oriented data analysis framework", Nuclear Instruments and Methods in Physics Reaserch A 389, 81 (1997). 